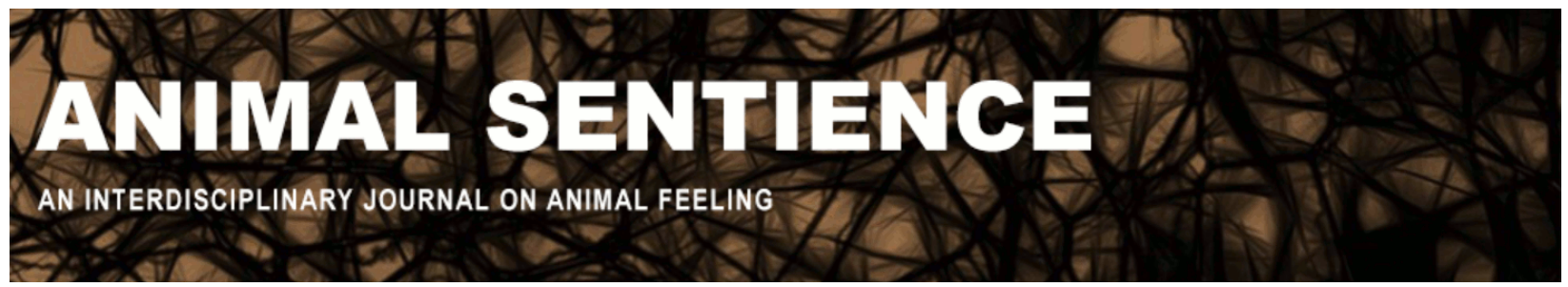

Damasio, Antonio and Damasio, Hanna (2016) Pain and other feelings in humans and animals. Animal Sentience 3(33)

DOI: $10.51291 / 2377-7478.1064$

Date of submission: 2015-11-12

Date of acceptance: 2015-12-13

(c)

This article has appeared in the journal Animal

Sentience, a peer-reviewed journal on animal

cognition and feeling. It has been made open access,

free for all, by WellBeing International and deposited

in the WBI Studies Repository. For more information,

please contact

wbisr-info@wellbeingintl.org.

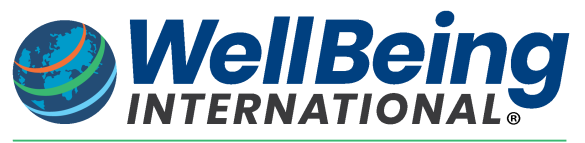

SOLUTIONS FOR PEOPLE, ANIMALS AND ENVIRONMENT 


\title{
Pain and other feelings in animals
}

Commentary on Key on Fish Pain

\author{
Antonio Damasio \& Hanna Damasio \\ Brain and Creativity Institute \\ University of Southern California, Los Angeles
}

\begin{abstract}
Evidence from neuroanatomy, neurophysiology, and neuropsychology suggests that the experience of feelings in humans does not depend exclusively on structures of the cerebral cortex. It does not seem warranted to deny the possibility of feeling in animals on the grounds that their cerebral cortices are not comparable to those of humans.
\end{abstract}

\begin{abstract}
Antonio Damasio damasio@dornsife.usc.edu is University Professor, David Dornsife Professor of Neuroscience, and Director of the Brain and Creativity Institute at the University of Southern California, and adjunct professor at the Salk Institute in La Jolla, California. He has made seminal contributions to the understanding of brain processes underlying, emotions,

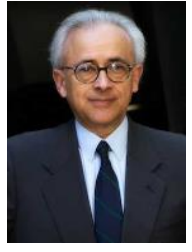
feelings, decision-making and consciousness as described in several books (Descartes' Error, The Feeling of What Happens, Looking for Spinoza, and Self Comes to Mind).

https://dornsife.usc.edu/cf/faculty-and-staff/faculty.cfm?pid=1008328
\end{abstract}

\begin{abstract}
Hanna Damasio hdamasio@college.usc.edu is University Professor, Dana Dornsife Professor of Neuroscience, and Director of the Dana and David Dornsife Cognitive Neuroscience Imaging Center at the University of Southern California. Using computerized tomography and magnetic resonance scanning, she has developed methods of investigating human brain structure and studied functions such as language, memory and emotion. She is the author of Lesion Analysis in Neuropsychology and Human Brain Anatomy in Computerized Images. http://ngp.usc.edu/faculty/profile/?fid=28
\end{abstract}

The stated purpose of Key's (2016) target article is to suggest that fish cannot feel pain. Toward this goal, Key uses a seemingly reasonable strategy: identify the neuroanatomical structures required to produce pain in humans, and ask whether other species, fish included, have a comparable "pain matrix" in their brains. Key argues that the cerebral cortex is indispensable for pain experience in humans, and because fish lack the corresponding structures, he concludes that fish cannot experience pain. On the way to reaching this conclusion, the author slides almost imperceptibly into a more general discussion of feelings (and, by extension, of consciousness), as if pain and other feelings were entirely comparable phenomenologically and had exactly the same underlying neurophysiology. While we have never investigated the status of pain or of other feelings in fish, we have been deeply interested in the equivalent functions of humans. In that respect, we are not convinced by Key's argument that 
pain in humans depends exclusively on the cerebral cortex. It may be the case that fish do not experience pain, but one cannot arrive at that conclusion by claiming that pain in humans is a pure consequence of cerebral cortex physiology.

Key argues that the experience of pain occurs in the brain, by which he means not the central nervous system, in general, or even the telencephalon, but exclusively the cerebral cortex. In favor of his conclusion, he argues that when the spinal cord is severed, the human ability to feel pain is lost below the level of the lesion, a sign that pain-related signals are transmitted from the body's periphery to the cerebral cortex. Key does not discuss the complex pain-related structures contained in the upper brain stem - the parabrachial nucleus, the nucleus tractus solitarius, and the periacqueductal gray, to name but a few - apparently assuming that they too are mere conduits to the cerebral cortex. They are not.

Key identifies a number of cortical structures as the main components of the pain matrix - the insular cortex, SI and SII, the cingulate cortex and certain prefrontal cortices - and also identifies certain key properties of the mammalian cerebral cortex as critical to the production of pain, namely, the ability to amplify signals and the ability to disseminate signals across varied cortical regions. Key also places great weight on the fact that electrical stimulation of structures belonging to the cortical pain matrix of humans would elicit pain. His description of the cortical pain matrix is standard. The problem comes with the interpretation of what the cortical matrix is presumed to accomplish and, importantly, with the fact that Key regards it as the exclusive provider of pain experience. We have serious reservations over this account for several reasons.

First, the distinctive aspect of any feeling is valence, the distinctive quality of pleasantness or unpleasantness, goodness or badness of the experience, which relate to how the current event is or is not conducive to a positive state of life regulation. We fail to see how the amplification and dissemination of signals in the cerebral cortex would explain the valence of pain or of feelings in general. It is quite likely that amplification and broad dissemination of pain-related signals have important cognitive consequences for the experience of pain. This is one of the specific roles that we have proposed for the insular cortex in the processing of any feeling (Damasio, 2010; Damasio et al., 2012; Damasio and Carvalho, 2013). But in all likelihood valence originates elsewhere, prior to the cortical level, built from a cascade of contributions made by the bloodstream (via critical nervous system regions devoid of the blood-brain-barrier), by peripheral nervous system pathways, and by subcortical nuclei in the brain stem, hypothalamus and basal forebrain.

Second, we do not see why cortical stimulation in humans would establish an exclusive role for the cerebral cortex in the experience of pain.

Third, Key justifiably calls attention to the fact that reflexes to nociceptive stimuli should not be interpreted as necessarily indicative of pain experiences. He argues that when fish appear to withdraw from a source of pain and fight against it, the behavior cannot be assumed to be a consequence of feeling pain. This is correct. But Key does not consider how such a reflex came to be selected in evolution. The fact remains that withdrawal responses are adaptive and work 
to counter dangerous developments from the point of view of homeostasis. We have no way of determining the degree to which, in animals, such simple responses would have been accompanied, evolutionarily, by an element of experience, that is, feeling; of note, the experience would not even need to be causative of the reaction. Also, there is no reason to assume that such an experiential component would require a mature cerebral cortex. It is sound to hypothesize, on the contrary, that some grade of experience could emerge from complex subcortical nuclei involved in homeostatic regulation and learning.

Fourth, our own observations in patients with complete bilateral destruction of insular cortices and intact feeling processes pose some problems for Key's definition of an exclusive cortical pain matrix in humans. Understandably, he wishes to reduce the significance of the findings. Key recognizes that in interpreting the findings in patient B we were, in his words, "reasonably circumspect in our conclusions." Indeed we were, exceptionally and perhaps unnecessarily circumspect, because we left open the possibility that since both SII and SI remained intact bilaterally, the patient's pain sensation could have been contributed by those structures. Still, our study of Patient B was not focused on pain but on feelings in general. It is unlikely that homeostatic feelings such as malaise or well-being, for example, have the same neurophysiological underpinnings as somatic pain. One does not normally refer such feelings to a specific body region, and the contributions of SII and SI to such experiences may be minor or non-existent. Also, Key's use of cases of unilateral insular damage and of thalamic pain to diminish the significance of our findings is problematic. For example, in a minority of cases of thalamic damage, pain is abolished. Key uses those few cases to suggest that the brain stem would fail to generate pain while ignoring the majority of thalamic cases in which pain is indeed present or even pathologically enhanced.

Of note, we have never denied the important contribution of the insular cortex to human feelings, pain included. On the contrary, we were among the first authors of the functionalimaging era to call attention to the role of the human insula in feelings of every sort, positive and negative, basic homeostatic or emotional (see Damasio et al., 2000, which Key does cite for a different purpose). The anatomical disproportion between the brain stem and the telencephalon, which is very marked in mammals and especially so in humans, is probably one reason why the utilization of feeling experiences in complex cognitive processes requires the agency of the insula. In other words, the insula is a cortical platform that allows communication of the subcortical feeling system with different cognitive- and motor-related cortices.

In conclusion, we do not see any evidence in favor of the idea that the engendering of feelings in humans would be confined to the cerebral cortex. On the contrary, based on anatomical and physiological evidence, subcortical structures and even the peripheral and enteric nervous systems appear to make important contributions to the experience of feelings. 


\section{References}

Damasio, A. (2010). Self Comes to Mind. New York, New York. Pantheon.

Damasio, A., \& Carvalho, G.B. (2013). The nature of feelings: evolutionary and neurobiological origins. Nature Reviews Neuroscience, 14(2), 143-152. doi:10.1038/nrn3403.

Damasio, A., Damasio, H., \& Tranel, D. (2012). Persistence of feelings and sentience after bilateral damage of the insula. Cerebral Cortex, 23(4), 833-846. doi:10.1093/cercor/bhs077.

Damasio, A.R., Grabowski, T.J., Bechara, A., Damasio, H., Ponto, L.L.B., Parvizi, J, \& Hichwa, R.D. (2000). Subcortical and cortical brain activity during the feeling of self-generated emotions. Nature Neuroscience, 3(10), 1049-1056. doi:10.1038/79871.

Key, B. (2016). Why fish do not feel pain. Animal Sentience 2016.003. 